\title{
Effect of Glucose or Fat Challenge on Aspirin Resistance in Diabetes
}

\author{
Hussein N. Yassine, ${ }^{1}$ Grace Davis-Gorman, ${ }^{2}$ Craig S. Stump, ${ }^{1,3}$ Stephen S. Thomson, ${ }^{1,3}$ \\ Justin Peterson, ${ }^{1}$ and Paul F. McDonagh ${ }^{2}$ \\ ${ }^{1}$ Division of Endocrinology, Department of Medicine, Arizona Health Sciences Center, University of Arizona, 1656 E Mabel Street, \\ Tucson, AZ 85724, USA \\ ${ }^{2}$ Cardiovascular and Thoracic Surgery and the Sarver Heart Center, Arizona Health Sciences Center, University of Arizona, \\ Tucson, AZ 85724, USA \\ ${ }^{3}$ Southern Arizona VA Health Care System, Tucson, AZ 85723, USA
}

Correspondence should be addressed to Hussein N. Yassine, hyassine@email.arizona.edu

Received 29 July 2010; Accepted 1 December 2010

Academic Editor: Dariush Elahi

Copyright (C) 2010 Hussein N. Yassine et al. This is an open access article distributed under the Creative Commons Attribution License, which permits unrestricted use, distribution, and reproduction in any medium, provided the original work is properly cited.

\begin{abstract}
Aspirin has lower antiplatelet activity in diabetic patients. Our aim is to study the roles of acute hyperglycemia and hyperlipidemia on aspirin function in diabetic subjects with and without cardiovascular disease. Using urine thromboxane (pg/mg creatinine) and VerifyNow (Aspirin Resistance Measures-ARU), we investigated diabetic subjects during a 2-hour glucose challenge $(n=49)$ or a 4-hour fat challenge $(n=11)$. All subjects were currently taking aspirin $(81 \mathrm{or} 325 \mathrm{mg})$. After fat ingestion, urine thromboxane increased in all subjects (Mean \pm SE before: after) $(1209 \pm 336: 1552 \pm 371, P=.01)$, while we noted a trend increase in VerifyNow measures $(408 \pm 8: 431 \pm 18, P=.1)$. The response to glucose ingestion was variable. Diabetic subjects with cardiac disease and dyslipidemia increased thromboxane $(1693 \pm 364: 2799 \pm 513, P<.05)$ and VerifyNow $(457.6 \pm 22.3: 527.1 \pm 25.8, P<.05)$ measures after glucose. We conclude that saturated fat ingestion increases in vivo thromboxane production despite aspirin therapy.
\end{abstract}

\section{Introduction}

Aspirin (ASA) is one of the most commonly used medications worldwide. There is no debate that ASA reduces mortality in secondary prevention of cardiovascular disease (CVD), but an important question is why some patients do not benefit from ASA therapy. It was suggested that diabetic patients not treated with aspirin therapy display increased platelet reactivity, and that aspirin treatment in diabetic patients is less effective than aspirin treatment in nondiabetic patients $[1,2]$. This phenomenon is known as aspirin resistance (ASA-R) [3]. ASA-R is not limited to diabetes and is documented in subjects with cardiovascular and kidney disease. Despite the absence of a current gold standard to define ASA resistance [4], urine thromboxane and VerifyNow point-of-care optical aggregometry are clinically validated with cutoff values that predict worse CVD outcomes [5-7]. Urine thromboxane is a measure on in vivo thromboxane production. We previously demonstrated that platelets are activated after incubation with increasing glucose concentrations in vitro [8]. Chronic hyperglycemia and hyperlipidemia are associated with ASA$\mathrm{R}$ [9], but the role of acute hyperglycemia and hyperlipidemia on ASA function in vivo has not been defined. In this study, we investigated the effects of acute glucose and fat ingestion on aspirin function in diabetic subjects with and without cardiovascular disease.

\section{Materials and Methods}

We studied 55 subjects with diabetes on ASA therapy for at least 2 weeks prior to testing. Subjects reported fasting to the Clinical and Translational Research Center (CATS) at 8 am. Blood was obtained on arrival and 1 hour after glucose $(n=49)$ or 4 hours after fat ingestion $(n=11)$. Five subjects 


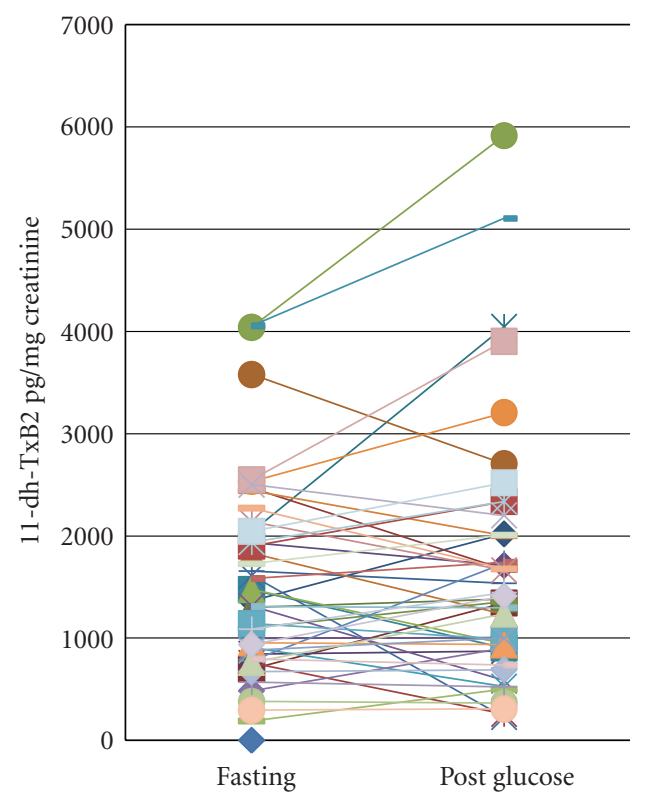

(a)

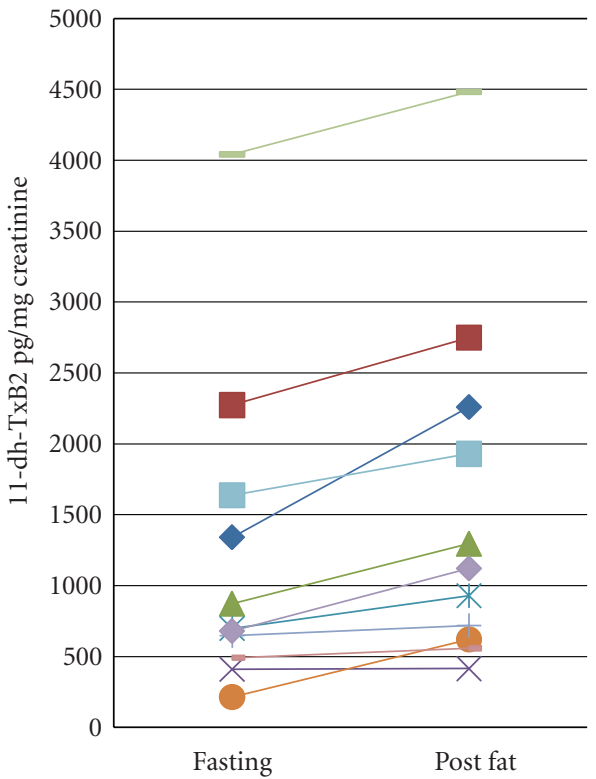

(b)

FIGURE 1: The response of urine thromboxane after glucose or fat ingestion (a). Urine thromboxane response to glucose ingestion $(n=49)$ (b). Urine thromboxane response to fat ingestion $(n=11)$. A more consistent increase in 11-dh-TxB2 was observed following the fat challenge compared to the glucose challenge. 11-dh-TxB2 = Thromboxane.

did both the fat and glucose challenge on separate days. The study was approved by our Institutional Review Board, and all patients provided written informed consent prior to testing. Clinical cardiovascular disease was defined by prior coronary artery bypass surgery (CABG), percutaneous transluminal angioplasty (PTCA), or thrombotic stroke. Patients were older than 18 years of age. The study excluded subjects if they met any of the following criteria: bleeding diathesis or a history of gastrointestinal bleeding, hemorrhagic stroke, illicit drug or alcohol abuse, coagulopathy, major surgery within 6 weeks prior to study, platelet count $<100,000 / \mathrm{mm}^{3}$, hematocrit $<25 \%$, creatinine $>4 \mathrm{mg} / \mathrm{dL}$, or current use of nonsteroidal anti-inflammatory drugs, anticoagulants, or antiplatelet drugs other than aspirin.

Blood and urine collection: after an overnight fast, blood was collected from the antecubital vein into Vacutainer tubes (Becton-Dickinson, Franklin Lakes, NJ) at 8 am to avoid a potential effect of circadian rhythm on platelet function. All patients were instructed to take their prescribed morning medications prior to blood draw. After collecting the first $2-3 \mathrm{~mL}$ of free flowing blood for other measurements, the tubes were filled to capacity and gently inverted 3 to 5 times to ensure complete mixing of the anticoagulant. Tubes containing 3.2\% sodium citrate (Greiner Bio-One Vacuette North America, Inc., Monroe, NC) were collected for VerifyNow measurements. Urine samples were collected for measurements of 11-dh-TxB2 (Corgenix, Bloomfield, $\mathrm{CO}$ ) and stored at $-20^{\circ} \mathrm{C}$ until analysis.

2.1. VerifyNow Aspirin Assay. VerifyNow (Accumetrics, CA) is a turbidimetric optical detection assay designed to measure platelet aggregation that is based upon the ability of activated platelets to bind to fibrinogen [6]. The cartridge contains a lyophilized preparation of human fibrinogen-coated beads, arachidonic acid, preservative, and buffer. The fibrinogencoated beads aggregate in whole blood in proportion to the number of unblocked platelet GPIIb/IIIa receptors. The instrument reports aggregation as aspirin resistance units (ARUs). There is no significant interaction between lipemia and VerifyNow measures as per the manufacturer. The coefficient of variance was $2.5 \%$ on repeated measures within patients. The between-patient coefficient of variance was $12.5 \%$ at baseline.

2.2. Urinary Thromboxane (11-dh-TxB2). AspirinWorks (Corgenix Co.) is an enzyme-linked immunosorbent assay (ELISA). In brief, assay buffer and urine were incubated with a monoclonal antibody followed by the addition of an 11-dh-TxB2-alkaline phosphate tracer. Urinary 11-dh-TxB2 concentrations were determined by measuring colorimetry at $405 \mathrm{~nm}$ using an ELISA reader and expressed as $\mathrm{pg} / \mathrm{mg}$ Creatinine $[1,7]$. The coefficient of variance was $4 \%$ on repeated measures within patients. The between-patient coefficient of variance was $14 \%$ at baseline.

2.3. Oral Glucose and Fat Meal. Forty-nine subjects ingested a standard glucose solution (Trutol 75, NERL Diagnostics) consisting of 75 grams of glucose. For the fat diet, 11 subjects ingested a standardized test meal [10] that consisted, per square meter of body surface area, of $175 \mathrm{~mL}$ of heavy whipped cream (Shamrock Farms, 99\% fat). Of 700 calories per square meter, $99 \%$ ( $70 \mathrm{~g}$ ) were derived from fat, $<1 \%$ was 
TABLE 1: Baseline measures.

\begin{tabular}{|c|c|}
\hline Patient characteristic & Diabetics $(n=56)$ \\
\hline Gender (M/F) & $28 / 28$ \\
\hline ASA dose $(81 \mathrm{mg} / 325 \mathrm{mg})$ & $48 / 8$ \\
\hline Prior CVD & $8 / 58(14 \%)$ \\
\hline Smoking, $n(\%)$ & $7 / 49(14 \%)$ \\
\hline Age (years) & $59 \pm 1.5$ \\
\hline BMI $\left(\mathrm{kg} / \mathrm{m}^{2}\right)$ & $37 \pm 3$ \\
\hline \multicolumn{2}{|l|}{ Medication (\%) } \\
\hline ACE inhibitors or ARB & $36 \%$ \\
\hline Statin & $51 \%$ \\
\hline Insulin & $43 \%$ \\
\hline Waist circumference $(\mathrm{cm})$ & $111 \pm 2$ \\
\hline Systolic BP (mmHg) & $130 \pm 2$ \\
\hline Diastolic BP (mmHg) & $78 \pm 1.2$ \\
\hline 11-dh-TxB2 (pg/mg creatinine) & $1465 \pm 125$ \\
\hline ASA-S (\%) & $64 \%$ \\
\hline VerifyNow (ARU) & $455 \pm 12$ \\
\hline ASA-S (\%) & $82 \%$ \\
\hline $\mathrm{LDL}(\mathrm{mg} / \mathrm{dL})$ & $105 \pm 5$ \\
\hline $\mathrm{HDL}(\mathrm{mg} / \mathrm{dL})$ & $47 \pm 2$ \\
\hline $\mathrm{TG}(\mathrm{mg} / \mathrm{dL})$ & $210 \pm 26$ \\
\hline Creatinine $(\mathrm{mg} / \mathrm{dL})$ & $0.8 \pm 0.04$ \\
\hline $\mathrm{HbAlc} \%$ & $8.5 \pm 0.3$ \\
\hline Fasting Glucose (mg/dL) & $169 \pm 11$ \\
\hline Duration of diabetes (years) & $10.5 \pm 1$ \\
\hline
\end{tabular}

Values are presented in means \pm SE.

11-dh-TxB2: Urine thromboxane, ARU: Aspirin resistance units, TG: Triglyceride, LDL: Low density lipoproteins, HDL: High density lipoprotein, ASA-S: Aspirin sensitive, ARB: Angiotensin receptor blocker, ACE: Angiotensin converting enzyme.

derived from carbohydrates $(<1 \mathrm{~g})$, or from proteins $(<1 \mathrm{~g})$. The cholesterol content was $25 \mathrm{mg}$, and the ratio of total polyunsaturated to saturated fat was 0.06 . We recorded ASA$\mathrm{R}$ measures at one and two hours after glucose ingestion, and two and four hours after fat ingestion. We report the most prominent changes that we noted at one hour after glucose ingestion and four hours after lipid ingestion. We measured weight, height, waist circumference, fasting lipids, HbAlc, uric acid, serum creatinine in all subjects at baseline, and glucose or triglycerides levels after ingestion of the glucose or fat challenge, respectively.

\subsection{Definitions}

2.4.1. Aspirin Resistance. Aspirin resistance was defined by previously reported criteria: $\geq 550$ ARU by VerifyNow [5] or $\geq 1500 \mathrm{pg} 11$-dh-TxB2/mg creatinine during treatment with $81 \mathrm{mg}$ or $325 \mathrm{mg}$ of daily aspirin [7]. We defined apriori a group of responders with change of at least $10 \%$ increase (calculated as premeasure/postmeasure) in thromboxane and $\mathrm{VN}$ response after glucose or fat challenge.
2.4.2. Statistical Analysis. Categorical variables were expressed as $n(\%)$ and continuous variables as mean \pm SE. Nonparametric data were log transformed. Kruskal Wallis was used to analyze groups with nonparametric distribution. A one-way analysis of variance (ANOVA) for repeated measures was used to compare levels of platelet function between and within diabetic and nondiabetic patients. An unpaired $t$-test was used to compare the prevalence of aspirin resistance between groups; $P<.05$ was considered statistically significant. Linear regression was used to explain the changes in measures of ASA-R to measures of glucose or triglyceride after the challenge. All statistical calculations were performed using SPSS software (Chicago, IL).

\section{Results}

Our study subjects were mostly Caucasian, middle-aged adults. The majority of our diabetic subjects were obese and had a history of hyperlipidemia, hypertension, and were receiving medical treatment such as statins, beta-blockers, and angiotensin converting enzyme inhibitors for prevention and treatment of CVD. Table 1 summarizes the baseline measurements.

The response to glucose or fat challenge is summarized in Table 2. Urine thromboxane was significantly increased in all 11 subjects after fat ingestion $(P=.01)$. Subjects demonstrated a variable response to the glucose ingestion (Figure 1). We did not find a correlation between the changes in glucose or triglyceride and the changes in 11-dh-TxB2 or VN. Within subjects that underwent the glucose challenge $(n=49)$, we had a mixed response to glucose ingestion (Figure 1(a)). We defined a group of subjects with at least $10 \%$ increase (calculated as premeasure/postmeasure) in thromboxane and $\mathrm{VN}$ response after glucose challenge (responders) and compared their profiles to subjects that did not change their measures of ASA$\mathrm{R}$ after the glucose challenge, using an independent $t$-test. In this analysis, 13 subjects did not change their 11-dhTxB2 measures, while 19 increased it by more than $10 \%$. For VN measures, 14 subjects did not change their ASA$\mathrm{R}$ measure, while 10 subjects had an increased response. We did not analyze the data from subjects that had a decrease in $\mathrm{TxB} 2$ or $\mathrm{VN}$ after the glucose ingestion. We $\log$ transformed the data to account for nonparametric distribution. As demonstrated in Table 3, subjects with an increased response to glucose were more aspirin resistant per 11-dh-TxB2 criteria at baseline compared to subjects where ASA-R measures did not change. Subjects with elevated 11dh-TxB2 or VN measures in response to glucose tended to have a prior history of CVD, greater LDL cholesterol, and longer duration of diabetes. There was no correlation between the change in plasma glucose before and after glucose ingestion and measures of aspirin resistance. After glucose ingestion, two subjects were defined as aspirin resistant per the urine thromboxane criteria and three subjects per VN criteria. After fat ingestion, one subject developed ASA-R using 11-dh-TxB2 cutoff values and one subject using $\mathrm{VN}$ values. There was no significant correlation 
TABLE 2: Response to glucose or fat challenge.

\begin{tabular}{|c|c|c|c|c|c|c|c|}
\hline \multicolumn{4}{|c|}{ Glucose challenge $(n=49)$} & & \multicolumn{3}{|c|}{ Fat challenge $(n=11)$} \\
\hline & Pre & Post & $\begin{array}{c}P \\
\text { value }\end{array}$ & & Pre & Post & $\begin{array}{c}P \\
\text { value }\end{array}$ \\
\hline Glucose $(\mathrm{mg} / \mathrm{dL})$ & $169 \pm 11$ & $289 \pm 16$ & $<.001$ & Triglyceride $(\mathrm{mg} / \mathrm{dL})$ & $210 \pm 26$ & $369 \pm 118$ & $<.001$ \\
\hline $\begin{array}{l}\text { 11-dh-TxB2 (pg/mg } \\
\text { creatinine) }\end{array}$ & $1538 \pm 131$ & $1806 \pm 247$ & .16 & $\begin{array}{l}11-\mathrm{dh}-\mathrm{TxB} 2(\mathrm{pg} / \mathrm{mg} \\
\text { creatinine })\end{array}$ & $1209 \pm 336$ & $1552 \pm 371$ & .01 \\
\hline VerifyNow (ARU) & $461 \pm 12$ & $453 \pm 13$ & .4 & VerifyNow (ARU) & $408 \pm 8$ & $431 \pm 18$ & .1 \\
\hline
\end{tabular}

Values are presented in means \pm SE.

TABle 3: Response to glucose ingestion.

\begin{tabular}{lcccc}
\hline & \multicolumn{2}{c}{11 -dh-TxB2 $(\mathrm{pg} / \mathrm{mg}$ of creatinine $)$} & \multicolumn{2}{c}{ VN (ARU) } \\
& Nonresponders $(n=13)$ & Responders $(n=19)$ & Nonresponders $(n=14)$ & Responders $(n=10)$ \\
\hline Pre-Glucose \% of ASA-R & $942 \pm 117$ & $1693 \pm 364^{\dagger}$ & $430.4 \pm 18.5$ & $457.6 \pm 22.3$ \\
& 15 & 55 & $428.5 \pm 18.1$ & 10 \\
Post-Glucose \% ASA-R & $954.1 \pm 122$ & $2799 \pm 513^{\dagger \dagger}$ & 7 & $527.1 \pm 25.8^{\ddagger}$ \\
CVD $(n)$ & 15 & 63 & 0 & 33 \\
Duration of diabetes & 1 & $4^{\dagger}$ & $4.9 \pm 1.4$ & $4^{\dagger}$ \\
LDL $(\mathrm{mg} / \mathrm{dL})$ & $6.3 \pm 1.7$ & $8.4 \pm 1.7$ & $105 \pm 9.4$ & $11.1 \pm 2.3^{\dagger}$ \\
\hline
\end{tabular}

Responders were defined by more than $10 \%$ increase in ASA-R measures after glucose ingestion. ASA-R: aspirin resistant, ASA-S: aspirin sensitive, CVD: cardiovascular disease.

Data are presented as means \pm SE. The following group comparisons denote $P<.05 .{ }^{\dagger}$ Non-responders: Responders, ${ }^{\ddagger}$ Pre: Post.

between the change in 11-dh-TxB2 / VN after glucose or fat ingestion.

\section{Discussion}

This is the first study to demonstrate that acute ingestion of saturated fat consistently increases in vivo thromboxane production, leading to a state of ASA-R. The lack of significance related to the measure of platelet function (VN) highlights that the fat load may have increased production/excretion of 11-dh-TxB2. Urinary thromboxane is not only a marker of platelet activity, but also a marker of inflammation and oxidative stress [11]. Urine thromboxane, as a global index of thromboxane synthesis, originates from other blood elements such as erythrocytes and monocytes and from renal biosynthesis [12]. We previously reported that whole blood incubation with increasing concentrations of glucose increased platelet function by the upregulation of P-selectin expression [8]. The current results suggest that the response to glucose ingestion in vivo is more complex. Subjects with chronic hyperglycemia, as assessed by the duration of diabetes, dyslipidemia, and prior CVD, are likely to be ASA$\mathrm{R}$ in response to a high glucose load. The state of increased thromboxane production and platelet activity after an acute fat or glucose load may be a mechanism explaining the role of a heavy meal as a trigger for acute myocardial infarction $[13,14]$.

Although there is no gold standard for defining ASA-R, definitions based on urine 11-dh-TxB2 and VN assays have cutoff values that are clinically validated to predict worse CVD outcomes. In a subanalysis of the Heart Outcomes
Prevention Evaluation (HOPE) study, the odds, over a 5 -year period, of the composite outcome of myocardial infarction, stroke, or CVD death in 976 ASA-treated patients (488 who suffered myocardial infarction, stroke, or CVD death and 488 sex- and age-matched controls) increased with each increasing quartile urine thromboxane levels [7]. The antiplatelet effect of aspirin measured by VerifyNow correlated with adverse clinical events in stable patients with $\mathrm{CAD}$ and is independent of known risk factors including diabetes and prior MI [5]. The prevalence of ASA-R in our study was $36 \%$ by urine $11-\mathrm{dh}-\mathrm{TxB} 2$, and $18 \%$ by $\mathrm{VN}$ measures (Table 1). In comparison, the prevalence of ASA resistance of diabetic subjects on $81 \mathrm{mg}$ of ASA in the ASPECT study was $37 \%$ by urine $11-\mathrm{dh}-\mathrm{TxB} 2$, and $13 \%$ for VN [1]. In another study [15] of subjects with CVD but not diabetes, ASA-R prevalence was $23 \%$ by 11 -dh-TxB2, and $7 \%$ by $\mathrm{VN}$.

Possible mechanism(s) for the reduced ability of ASA to sufficiently protect against intravascular occlusions in diabetes mellitus (DM) patients compliant with ASA therapy include chronic hyperglycemia, hyperlipidemia, and suboptimal ASA dosing, particularly in relation to altered platelet turnover in diabetic patients $[16,17]$. The activating role of chronic hyperlipidemia on platelet function was demonstrated both in vivo [18] and in vitro [19]. Friend et al. [18] found that platelet responsiveness to aspirin is reduced in patients with chronic hyperlipidemia. Davi et al. [20] observed enhanced in vivo formation of the F2isoprostane 8-epi-PGF2a in chronic hyperlipidemia that correlated with enhanced urine $11-\mathrm{dh}-\mathrm{TxB} 2$ production. This provides an aspirin-insensitive mechanism possibly linking lipid peroxidation to amplification of platelet activation. 
Moreover, platelet responsiveness to ASA improves after statin therapy [21]. Alternatively, oxidized LDL and oxidized choline glycerophospholipids induce platelet activation and thrombosis in fat fed mice through platelet CD36 [19]. The molecular mechanisms explaining platelet response to acute glucose loads might be more complex. We suggest that rather than acute hyperglycemia per se, it is the mishandling of hyperglycemia and resulting libration of oxygen-free radicals [22] in subjects with chronic diabetes, dyslipidemia, and prior cardiovascular disease leading to increased platelet activation and thromboxane production.

The limitations of this study are the small sample size, the absence of a control group, and a CVD cohort without diabetes. The acute response to glucose or fat ingestion may not reflect the chronic condition. Although we noted a trend increase in VerifyNow after fat ingestion, the lack of a significant change is possibly related to our small cohort size. The greater variability in the prechallenge 11-dh-TxB2 between the glucose and fat groups is likely related to the small size of the fat challenge group.

\section{Conclusion}

In summary, our study indicates that saturated fat in the diet increases in vivo thromboxane production. This highlights the importance of low dietary saturated fat intake and aggressive lipid management in diabetic patients on ASA therapy.

\section{Conflict of Interests}

The authors have no conflict of interests to disclose.

\section{Acknowledgments}

The authors are indebted to Kirk Guyer and Corgenix staff for support with urine thromboxane kits, and Ji-eun Choi for her assistance in performing the ELISA assays. The authors thank Chris Wendell for his statistical support, and Eric Schwartz for the assistance with the fat challenge protocol, and Karen Grinfeld for reviewing the manuscript. This work was supported by NIH HLB 58859 (P. F. McDonagh), State of Arizona TRIF Fund (C. S. Stump), and Veteran Affairs Career Development Grant (C. S. Stump).

\section{References}

[1] J. DiChiara, K. P. Bliden, U. S. Tantry et al., "The effect of aspirin dosing on platelet function in diabetic and nondiabetic patients: an analysis from the aspirin-induced platelet effect (ASPECT) study," Diabetes, vol. 56, no. 12, pp. 3014-3019, 2007.

[2] C. Watala, "Blood platelet reactivity and its pharmacological modulation in (people with) diabetes mellitus," Current Pharmaceutical Design, vol. 11, no. 18, pp. 2331-2365, 2005.

[3] T. H. Wang, D. L. Bhatt, and E. J. Topol, "Aspirin and clopidogrel resistance: an emerging clinical entity," European Heart Journal, vol. 27, no. 6, pp. 647-654, 2006.
[4] M. Lordkipanidzé, C. Pharand, D. A. Palisaitis, and J. G. Diodati, "Aspirin resistance: truth or dare," Pharmacology and Therapeutics, vol. 112, no. 3, pp. 733-743, 2006.

[5] W. H. Chen, XI. Cheng, P. Y. Lee et al., "Aspirin resistance and adverse clinical events in patients with coronary artery disease," American Journal of Medicine, vol. 120, no. 7, pp. 631635, 2007.

[6] W. H. Chen, P. Y. Lee, W. Ng, H. F. Tse, and C. P. Lau, "Aspirin resistance is associated with a high incidence of myonecrosis after non-urgent percutaneous coronary intervention despite clopidogrel pretreatment," Journal of the American College of Cardiology, vol. 43, no. 6, pp. 1122-1126, 2004.

[7] J. W. Eikelboom, J. Hirsh, J. I. Weitz, M. Johnston, Q. Yi, and S. Yusuf, "Aspirin-resistant thromboxane biosynthesis and the risk of myocardial infarction, stroke, or cardiovascular death in patients at high risk for cardiovascular events," Circulation, vol. 105, no. 14, pp. 1650-1655, 2002.

[8] A. Le Guyader, G. Pacheco, N. Seaver, G. Davis-Gorman, J. Copeland, and P. F. McDonagh, "Inhibition of platelet GPIIbIIIa and P-selectin expression by aspirin is impaired by stress hyperglycemia," Journal of Diabetes and Its Complications, vol. 23, no. 1, pp. 65-70, 2009.

[9] C. Watala, J. Golanski, J. Pluta et al., "Reduced sensitivity of platelets from type 2 diabetic patients to acetylsalicylic acid (aspirin)-its relation to metabolic control," Thrombosis Research, vol. 113, no. 2, pp. 101-113, 2004.

[10] A. J. H. H. M. V. Oostrom, T. J. Rabelink, C. Verseyden et al., "Activation of leukocytes by postprandial lipemia in healthy volunteers," Atherosclerosis, vol. 177, no. 1, pp. 175-182, 2004.

[11] G. Davì, M. T. Guagnano, G. Ciabattoni et al., "Platelet activation in obese women: role of inflammation and oxidant stress," Journal of the American Medical Association, vol. 288, no. 16, pp. 2008-2014, 2002.

[12] H. A. Tran, S. S. Anand, G. J. Hankey, and J. W. Eikelboom, "Aspirin resistance," Thrombosis Research, vol. 120, no. 3, pp. 337-346, 2007.

[13] R. A. Kloner, "The "merry christmas coronary" and "happy new year heart attack" phenomenon," Circulation, vol. 110, no. 25, pp. 3744-3745, 2004.

[14] T. M. Kolettis, A. Papathanasiou, D. Tziallas, H. J. Milionis, C. K. Kastanioti, and K. Achenbach, "Afternoon nap, meal ingestion and circadian variation of acute myocardial infarction," International Journal of Cardiology, vol. 123, no. 3, pp. 338340, 2008.

[15] M. Lordkipanidzé, C. Pharand, E. Schampaert, J. Turgeon, D. A. Palisaitis, and J. G. Diodati, "A comparison of six major platelet function tests to determine the prevalence of aspirin resistance in patients with stable coronary artery disease," European Heart Journal, vol. 28, no. 14, pp. 1702-1708, 2007.

[16] D. Tschoepe, P. Roesen, J. Esser et al., "Large platelets circulate in an activated state in diabetes mellitus," Seminars in Thrombosis and Hemostasis, vol. 17, no. 4, pp. 433-438, 1991.

[17] P. D. Winocour, "Platelet turnover in advanced diabetes," European Journal of Clinical Investigation, vol. 24, Supplement 1, pp. 34-37, 1994.

[18] M. Friend, I. Vucenik, and M. Miller, "Platelet responsiveness to aspirin in patients with hyperlipidaemia," British Medical Journal, vol. 326, no. 7380, pp. 82-83, 2003.

[19] E. A. Podrez, T. V. Byzova, M. Febbraio et al., "Platelet CD36 links hyperlipidemia, oxidant stress and a prothrombotic phenotype," Nature Medicine, vol. 13, no. 9, pp. 1086-1095, 2007. 
[20] G. Davi, P. Alessandrini, A. Mezzetti et al., "In vivo formation of 8-epi-prostaglandin $\mathrm{F}(2 \alpha)$ is increased in hypercholesterolemia," Arteriosclerosis, Thrombosis, and Vascular Biology, vol. 17, no. 11, pp. 3230-3235, 1997.

[21] M. Labiós, M. Martínez, F. Gabriel, V. Guiral, E. Martínez, and J. Aznar, "Effect of atorvastatin upon platelet activation in hypercholesterolemia, evaluated by flow cymetry," Thrombosis Research, vol. 115, no. 4, pp. 263-270, 2005.

[22] J. F. Keaney and J. Loscalzo, "Diabetes, oxidative stress, and platelet activation,” Circulation, vol. 99, no. 2, pp. 189-191, 1999. 


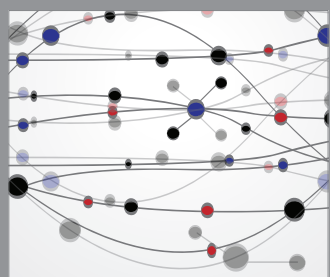

The Scientific World Journal
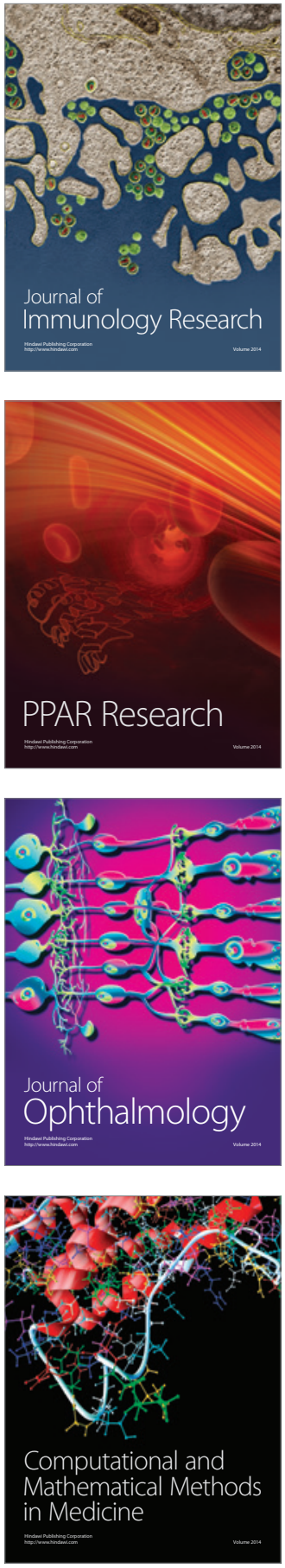

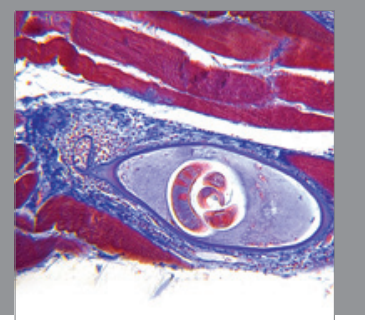

Gastroenterology

Research and Practice
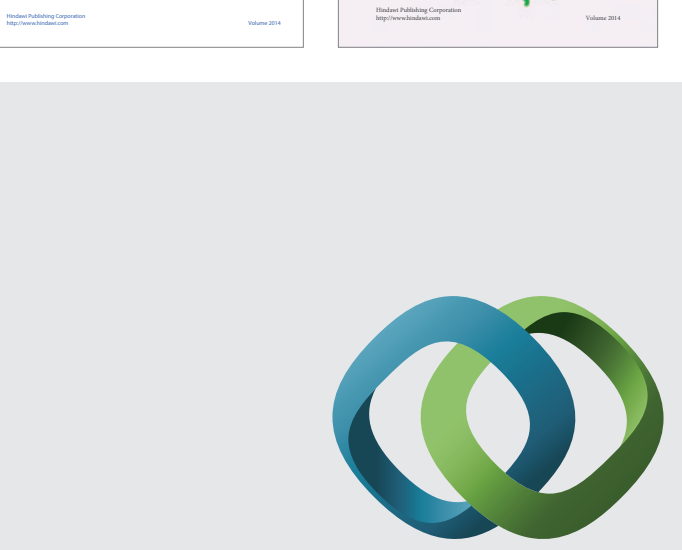

\section{Hindawi}

Submit your manuscripts at

http://www.hindawi.com
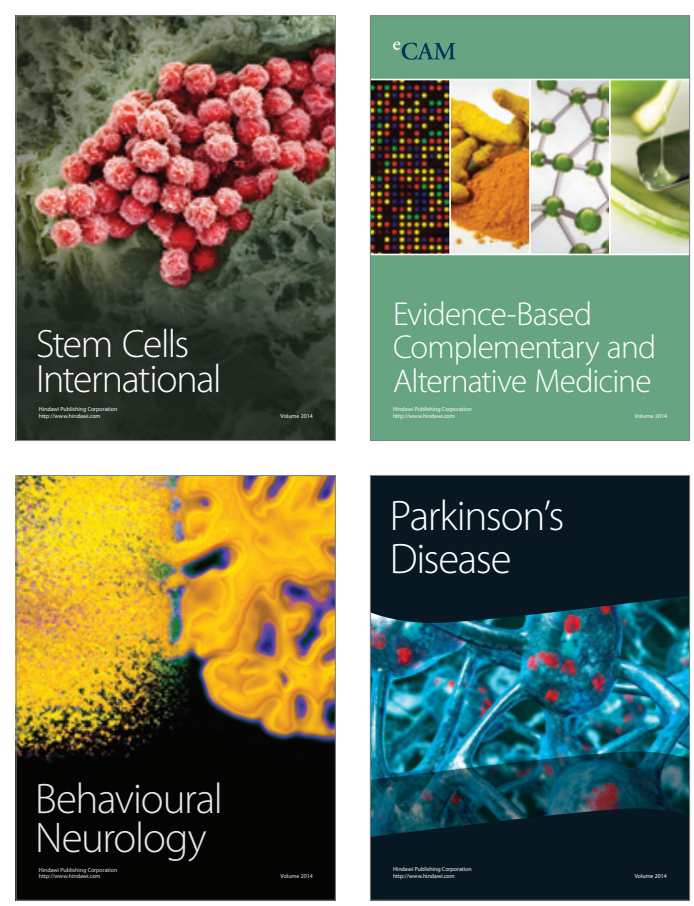

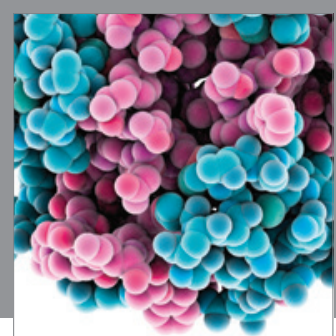

Journal of
Diabetes Research

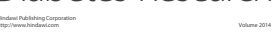

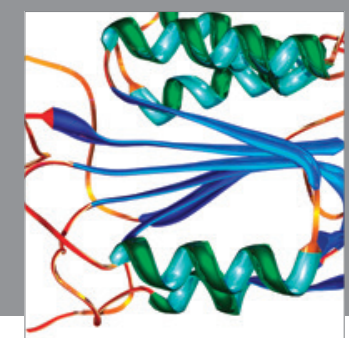

Disease Markers
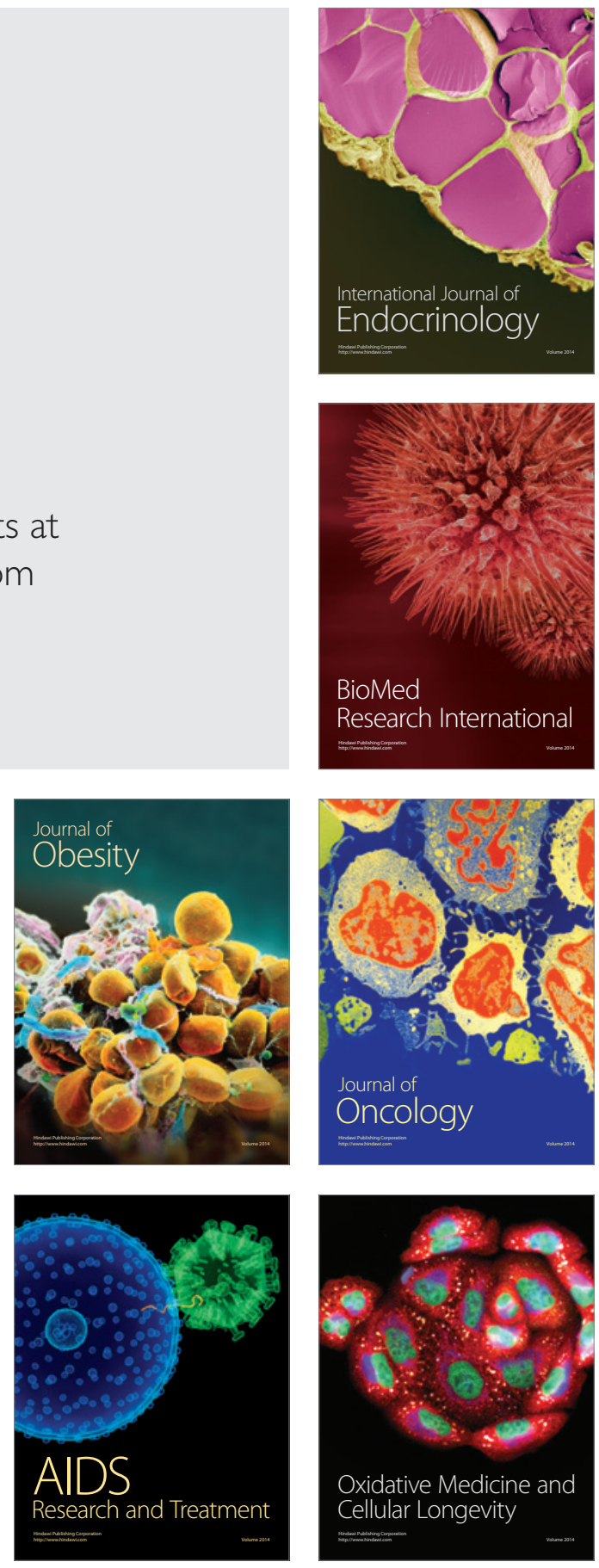